\title{
Standard Deviation of the Run Length (SDRL) and Average Run Length (ARL) Performances of EWMA and Synthetic Charts
}

\author{
Michael B. C. Khoo, S. Y. Teh, X. Y. Chew, and W. L. Teoh
}

\begin{abstract}
Statistical process control (SPC) techniques are applied to monitor a process. The control chart is a valuable tool in SPC. the shewhart control chart was the first chart proposed in the literature of SPC and it is still used in process monitoring in today's manufacturing and service industries. both the exponentially weighted moving average (EWMA) and synthetic charts outperform the shewhart control chart for detecting shifts in the process mean. Since both the EWMA and synthetic charts provide better process mean shifts detection performance, a study on which chart to use in process monitoring under different situations is the aim of this work. this paper compares the average run length (ARL) and standard deviation of the run length (SDRL) profiles of the EWMA and synthetic charts. comparisons are made based on the normality assumption. The mathematica programs are used to compute the ARLs and SDRLs of the EWMA and synthetic charts. the ARL results indicate that the EWMA chart is superior to the synthetic chart for detecting small mean shifts, but the latter prevails for detecting moderate and large shifts. however, in terms of the SDRL, the EWMA chart surpasses the synthetic chart for small and moderate shifts.
\end{abstract}

Index Terms-Average run length (ARL), exponentially weighted moving average (EWMA) chart, synthetic chart, standard deviation of the run length (SDRL).

\section{INTRODUCTION}

The control chart which was originally developed at the Bell Laboratories by Dr Walter Shewhart [1] in 1924 is one of the primary tools in SPC. Since then, numerous research works were made on various types of control charts which include univariateand multivariate charts [2]. Two of the univariate charts considered in this paper are the EWMA and synthetic charts. The performance of a control chart is typically measured in terms of the ARL and SDRL. The ARL is the average number of sample points that is plotted on a chart before the first out-of-control signal is detected whereas the SDRL measures the spread of the run length distribution. When a process is out-of-control, it is desirable to have small values of $A R L$ and SDRL. Sections II and III review the EWMA and synthetic charts, respectively. Sections IV and V studies the ARL and SDRL performances

Manuscript received May 14, 2014; revised August 17, 2014. This research is supported by the Universiti Sains Malaysia (USM), Fundamental Research Grant Scheme (FRGS), no. 203/PMATHS/6711322.

Michael B. C. Khoo and X. Y. Chew are with the School of Mathematical Sciences, Universiti Sains Malaysia, CO 11800 Malaysia (email: mkbc@usm.my; chewxying@gmail.com).

S. Y. Teh is with the School of Management, Universiti Sains Malaysia, CO 11800 Minden, Penang, Malaysia (e-mail: tehsyin@usm.my).

W. L. Teoh is with the Department of Physical and Mathematical Science, Faculty of Science, Universiti Tunku Abdul Rahman, CO 31900 Malaysia (e-mail: teohwl@utar.edu.my). of the EWMA and synthetic charts, respectively, whereas Section VI compares the ARL and SDRL performances of these two charts. Finally, conclusions are drawn in Section VII.

\section{EWMA CHART}

The EWMA chart was introduced by Roberts [3] and further studied by Crowder [4]-[6], and Lucas and Saccucci [7]. The EWMA chart is a good alternative to the Shewhart $\bar{X}$ chart for detecting small shifts [2], [3]. The EWMA statistic is defined as follows:

$$
Z_{i}=\lambda \bar{X}_{i}+(1-\lambda) Z_{i-1}
$$

where $0<\lambda \leq 1$ is thesmoothing constant. The starting value is the process target, i.e. $Z_{0}=\mu_{0}$. The control limits $(U C L / L C L)$ and center line $(C L)$ for the EWMA chart are defined as follows [2], [3]:

$$
\begin{gathered}
U C L=\mu_{0}+L \sigma \sqrt{\frac{\lambda}{n(2-\lambda)}\left[1-(1-\lambda)^{2 i}\right]}, \\
C L=\mu_{0}, \text { and } \\
L C L=\mu_{0}-L \sigma \sqrt{\frac{\lambda}{n(2-\lambda)}\left[1-(1-\lambda)^{2 i}\right]},
\end{gathered}
$$

where the factor $L$ controls the width of the control limits. An out-of-control is signaled by the EWMA chart when $Z_{i}$ plots beyond the limits $U C L / L C L$.In this paper, the ARL of the EWMA chart is computed using the Markov chain approach presented in Zhang et al. [8]. The Markov chain procedure involves dividing the interval between the $U C L$ and $L C L$ into $t=2 m+1$ subintervals, each of width $2 d$. The EWMA statistic, $Z_{i}$ is said to be in a transient state $j$ at time $i$ if $S_{j}-d<Z_{i}<S_{j}+d$, for $j=-m,-m+1, \ldots, 0$, $\ldots, m-1, m$, where $S_{j}$ represents the midpoint of the $j^{\text {th }}$ subinterval. The run length of the EWMA chart is represented by its initial probability vector and transition probability matrix. The initial probability vector is

$$
\boldsymbol{u}=(0, \ldots, 0,1,0, \ldots 0)^{\prime}
$$

Here, the entry having the value unity corresponds to the initial state of the Markov chain, where the $(m+1)^{\text {th }}$ entry of $\boldsymbol{u}$ has the value unity.

The ARL of the EWMA chart is computed as [8] 


$$
\mathrm{ARL}=\boldsymbol{u}^{\prime}(\mathbf{I}-\boldsymbol{R})^{-\mathbf{1}} \mathbf{1}
$$

while its SDRL is obtained using the following formula:

$$
\mathrm{SDRL}=\sqrt{2 \mathbf{u}^{\prime}(\mathbf{I}-\boldsymbol{R})^{-2} \boldsymbol{R} \cdot \mathbf{1}-(\mathrm{ARL})^{2}+\mathrm{ARL}},
$$

where denotes a vector of ones, $\boldsymbol{I}$ represents the identity matrix whereas $\boldsymbol{R}$ is the transition probability matrix for the transient states. The details for computing $\boldsymbol{R}$ is given in Zhang et al. [8].

\section{SYNTHETIC CHART}

The synthetic chart which comprises the $\bar{X} / S$ and $\mathrm{CRL} / \mathrm{S}$ sub-charts was suggested by $\mathrm{Wu}$ and Spedding [9]. The implementation of the synthetic chart is as follows:

Step 1: Compute the $\bar{X} / S$ sub-chart's upper and lower control limits as follows:

$$
U C L_{\overline{\mathbf{x}} / \mathbf{s}}=\mu+k \sigma_{\overline{\mathbf{x}}},
$$

and

$$
L C L_{\overline{\mathbf{x}} / \mathbf{s}}=\mu-k \sigma_{\overline{\mathbf{x}}},
$$

where $\mu$ and $\sigma_{\bar{X}}$ are the in-control mean and standard deviation of the sample mean $\bar{X}$, respectively. The factor $k$ controls the width of the control limits.

Step 2: Compute the $C R L / \mathrm{S}$ sub-chart's lower control limit, $L^{\prime}$.

Step 3: At each inspection point, take a random sample of $n$ observations, $X_{i}$, for $i=1,2, \ldots, n$, and calculate the sample mean, $\bar{X}$.

Step 4: If $L C L_{\bar{X} / S}<\bar{X}<U C L_{\bar{X} / S}$, the sample is conforming and the control flow returns to Step 3. Otherwise, the sample is non-conforming and the control flow goes to the next step.

Step 5: Count the number of samples between the current and the last non-conforming samples. This number is taken as the CRL value of the CRL/S sub-chart.

Step 6: If $C R L>L^{\prime}$, the process is in-control and the control flow goes back to Step 3. Otherwise, the synthetic chart signals an out-of-control condition. Corrective actions are taken to identify and remove the assignable cause(s). Then the control flow returns to Step 3.

The $A R L$ of the synthetic chart is computed as follows:

$$
\mathrm{ARL}=\frac{1}{P} \times \frac{1}{1-(1-P)^{L^{L}}},
$$

where

$$
P=1-[(k-\delta \sqrt{n})-(-k-\delta \sqrt{n})] .
$$

Here, $\delta$ is the size of the standardized mean shift, i.e. $\delta=\frac{\left|\mu_{1}-\mu_{0}\right|}{\sigma}$, where $\mu_{1}$ is the out-of-control mean and $\mu_{0}$ is the in-control mean. When $\delta=0$, the process is incontrol.

\section{ARL AND SDRL PERFoRMANCES OF THE EWMA CHART}

The optimal parameters $\lambda$ and $L$ are obtained by minimizing the out-of-control ARL for a desired shift of interest $\delta_{\text {opt }}=1$ so that an in-control ARL $\left(\mathrm{ARL}_{0}\right)$ of 370 is attained, based on the sample sizes $n=3,5,7$, and 10 . The optimal design procedure is based on the Markov chain approach described in Zhang et al. [8]. From these optimal $\lambda$ and $L$ values obtained, the ARL profiles for the entire magnitude of shifts can be computed.

TABLE I: ARLS AND SDRLS OF THE EWMA CHART FOR $n=3,5,7$, AND 10, WHEN $\delta_{\text {opt }}=1$, BASED ON $\mathrm{ARL}_{0}=370$

\begin{tabular}{ccccccccc}
\hline \hline$n$ & 3 & 5 & 7 & 10 & 3 & 5 & 7 & 10 \\
$\lambda$ & 0.31 & 0.44 & 0.59 & 0.75 & 0.31 & 0.44 & 0.59 & 0.75 \\
$L$ & 0.735 & 0.715 & 0.731 & 0.734 & 0.735 & 0.715 & 0.731 & 0.734 \\
\hline$\delta$ & \multicolumn{6}{c}{ ARL } \\
\cline { 2 - 8 } 0.00 & 371.9 & 370.2 & 372.5 & 372.9 & 369.1 & 368.4 & 371.2 & 369.7 \\
0.30 & 63.4 & 50.8 & 48.6 & 16.8 & 59.8 & 48.2 & 46.8 & 13.1 \\
0.50 & 14.9 & 10.6 & 9.1 & 4.8 & 11.6 & 8.3 & 7.4 & 2.3 \\
0.80 & 6.7 & 4.6 & 3.6 & 2.8 & 4.0 & 2.7 & 2.3 & 1.0 \\
1.00 & 4.2 & 2.8 & 2.2 & 2.0 & 2.0 & 1.3 & 1.1 & 0.6 \\
1.50 & 2.4 & 1.7 & 1.3 & 1.3 & 0.8 & 0.6 & 0.5 & 0.5 \\
2.00 & 1.8 & 1.2 & 1.0 & 1.0 & 0.6 & 0.4 & 0.1 & 0.1 \\
3.00 & 1.1 & 1.0 & 1.0 & 1.0 & 0.3 & 0.0 & 0.0 & 0.0 \\
4.00 & 1.0 & 1.0 & 1.0 & 1.0 & 0.0 & 0.0 & 0.0 & 0.0 \\
\hline \hline
\end{tabular}

Table I shows the ARL and SDRL profiles for $0 \leq \delta \leq 4$. It is obvious that both ARL and SDRL decrease as $\delta$ increases. This indicates that larger shifts can be detected quicker and will result in smaller spread in the run length distribution. The results also show that as the sample size increases from $n=3$ to $n=10$, the ARLs and SDRLs of the EWMA chart reduce, for the same size of a mean shift, $\delta$.

\section{ARL AND SDRL PERFORMANCES OF THE SYNTHETIC CHART}

TABLE II: ARLS AND SDRLS OF THE SYNTHETIC CHART FOR $n=3,5,7$, AND 10, WHEN $\delta_{\text {opt }}=1$, BASED ON $\mathrm{ARL}_{0}=370$

\begin{tabular}{ccccccccc}
\hline \hline$n$ & 3 & 5 & 7 & 10 & 3 & 5 & 7 & 10 \\
$L^{\prime}$ & 6 & 4 & 3 & 2 & 6 & 4 & 3 & 2 \\
$k$ & 2.294 & 2.219 & 2.164 & 2.085 & 2.294 & 2.219 & 2.164 & 2.085 \\
\hline$\delta$ & \multicolumn{6}{c}{ ARL } \\
\cline { 2 - 9 } 0.00 & 370.0 & 370.0 & 370.0 & 370.0 & 409.8 & 403.2 & 399.2 & 394.2 \\
0.50 & 33.6 & 16.6 & 10.2 & 6.3 & 41.8 & 20.7 & 12.6 & 7.5 \\
0.75 & 9.6 & 4.5 & 2.9 & 1.9 & 12.1 & 5.3 & 3.0 & 1.8 \\
1.00 & 4.0 & 2.1 & 1.5 & 1.2 & 4.4 & 1.8 & 1.0 & 0.6 \\
1.50 & 1.6 & 1.1 & 1.0 & 1.0 & 1.0 & 0.4 & 0.2 & 0.1 \\
2.00 & 1.1 & 1.0 & 1.0 & 1.0 & 0.4 & 0.1 & 0.0 & 0.0 \\
3.00 & 1.0 & 1.0 & 1.0 & 1.0 & 0.0 & 0.0 & 0.0 & 0.0 \\
4.00 & 1.0 & 1.0 & 1.0 & 1.0 & 0.0 & 0.0 & 0.0 & 0.0 \\
\hline \hline
\end{tabular}

To ensure a fair comparison, the optimal $L^{\prime}$ and $k$ values of the synthetic chart are determined so that $\mathrm{ARL}_{0}=$ $370, \delta_{\text {opt }}=1$ and $n=3,5,7$, and 10. The optimization procedure given in $\mathrm{Wu}$ and Spedding [9] is used to compute the optimal parameters $L^{\prime}$ and $k$. Then using the optimal parameters $L^{\prime}$ and $k$, the ARL of the synthetic chart is computed using (7a) and (7b), while its SDRL is obtained 
using (5) but by substituting $\boldsymbol{u}, \boldsymbol{R}$, and ARL with that corresponding to the synthetic chart [10].

For example, for $\mathrm{ARL}_{0}=370, \delta_{\text {opt }}=1$, and $n=3$, the optimal parameters of the synthetic chart are computed as $L^{\prime}=6$ and $k=2.294$. Table II indicates that as $\delta$ increases (size of a mean shift increases), the ARLs and SDRLs decrease. The findings also show that as the sample size, $n$, increases, the ARLs and SDRLs decrease for the same magnitude of a mean shift, $\delta$.

VI. A COMPARISON OF THE ARL AND SDRL PERFORMANCES OF THE EWMA AND SYNTHETIC CHARTS

TABLE III: ARLS OF THE EWMA AND SYNTHETIC CHARTS, WHERE $n=3$, 5,7, AND $10, \delta_{\text {opt }}=1, \mathrm{AND} \mathrm{ARL}_{0}=370$

\begin{tabular}{ccccccccc}
\hline \hline$n$ & 3 & 5 & 7 & 10 & 3 & 5 & 7 & 10 \\
\hline$\delta$ & \multicolumn{3}{c}{ EWMA } & \multicolumn{5}{c}{ Synthetic } \\
\cline { 2 - 9 } 0.00 & 371.9 & 370.2 & 372.5 & 372.9 & 370.0 & 370.0 & 370.0 & 370.0 \\
0.25 & 63.4 & 50.8 & 48.6 & 16.8 & 151.8 & 102.8 & 75.4 & 52.8 \\
0.50 & 14.9 & 10.6 & 9.1 & 4.8 & 33.6 & 16.6 & 10.2 & 6.3 \\
0.75 & 6.7 & 4.6 & 3.6 & 2.8 & 9.6 & 4.5 & 2.9 & 1.9 \\
1.00 & 4.2 & 2.8 & 2.2 & 2.0 & 4.0 & 2.1 & 1.5 & 1.2 \\
1.50 & 2.4 & 1.7 & 1.3 & 1.3 & 1.6 & 1.1 & 1.0 & 1.0 \\
2.00 & 1.8 & 1.2 & 1.0 & 1.0 & 1.1 & 1.0 & 1.0 & 1.0 \\
3.00 & 1.1 & 1.0 & 1.0 & 1.0 & 1.0 & 1.0 & 1.0 & 1.0 \\
4.00 & 1.0 & 1.0 & 1.0 & 1.0 & 1.0 & 1.0 & 1.0 & 1.0 \\
\hline \hline
\end{tabular}

TABLE IV: SDRLS OF THE EWMA AND SYNTHETIC CHARTS, WHERE $n=$ $3,5,7$, AND $10, \delta_{\text {opt }}=1, \mathrm{AND} \mathrm{ARL}_{0}=370$

\begin{tabular}{ccccccccc}
\hline \hline$n$ & 3 & 5 & 7 & 10 & 3 & 5 & 7 & 10 \\
\hline$\delta$ & \multicolumn{3}{c}{ EWMA } & \multicolumn{5}{c}{ Synthetic } \\
\cline { 2 - 9 } 0.00 & 369.1 & 368.4 & 371.2 & 369.7 & 409.8 & 403.2 & 399.2 & 394.2 \\
0.25 & 59.8 & 48.2 & 46.8 & 13.1 & 175.0 & 118.3 & 86.6 & 60.3 \\
0.50 & 11.6 & 8.3 & 7.4 & 2.3 & 41.8 & 20.7 & 12.6 & 7.5 \\
0.75 & 4.0 & 2.7 & 2.3 & 1.0 & 12.1 & 5.3 & 3.0 & 1.8 \\
1.00 & 2.0 & 1.3 & 1.1 & 0.6 & 4.4 & 1.8 & 1.0 & 0.6 \\
1.50 & 0.8 & 0.6 & 0.5 & 0.5 & 1.0 & 0.4 & 0.2 & 0.1 \\
2.00 & 0.6 & 0.4 & 0.1 & 0.1 & 0.4 & 0.1 & 0.0 & 0.0 \\
3.00 & 0.3 & 0.0 & 0.0 & 0.0 & 0.0 & 0.0 & 0.0 & 0.0 \\
4.00 & 0.0 & 0.0 & 0.0 & 0.0 & 0.0 & 0.0 & 0.0 & 0.0 \\
\hline \hline
\end{tabular}

Combining the ARLs and SDRLs in Table I and Table II give the results in Table III and Table IV. Table III clearly shows that the ARL produced by the EWMA chart is smaller than that of the synthetic chart when $0<\delta \leq 0.75$. However, the synthetic chart gives smaller ARL than the EWMA chart when $\delta \geq 1$. Table IV shows that the EWMA chart outperforms the synthetic chart for $\delta \leq 1.5$, in terms of the SDRL. The ARL results show that the EWMAchart is superior to the synthetic chart for detecting small mean shifts, but the latter prevails for detecting moderate and large shifts. However, in terms of the SDRL, the EWMA chart surpasses the synthetic chart for small and moderate $\delta$ values. This study guides industrial practitioners to choose between the EWMA and synthetic charts. For example, if past experience indicates that a small mean shift is likely to occur, then the EWMA chart should be used to monitor the process. However, if historical data show that a moderate or large shift usually happens, then the synthetic chart should be employed in process monitoring. Overall, similar trends are observed for $n=3,5,7$, and 10, as displayed in Table III to Table IV, respectively. It should be noted that considering other combinations of $\delta_{\mathrm{opt}}$ and $\mathrm{ARL}_{0}$ will give similar outcomes. Therefore, it suffices to only discuss the results for $\delta_{\text {opt }}=1, \mathrm{ARL}_{0}=370$, and $n=$ $3,5,7$ and 10. The results for $\delta_{\text {opt }}=1, \mathrm{ARL}_{0}=250$, and $n$ $=3,5,7$ and 10 which correspond to Tables I to IV are given in the Appendix (see Tables A.I to A.IV).

\section{CONCLUSION}

In this paper, the ARLs and SDRLs of the EWMA and synthetic charts are compared for a process having a normal distribution. This preliminary study provides some insight on EWMA and synthetic charts to practitioners to facilitate process monitoring, where a proper and careful selection of the correct chart to use is of utmost importance so that a delay in detecting process shifts will not happen. Further research should compare the EWMA and synthetic charts using other performance criteria, such as the median run length (MRL) and percentiles of the run length distribution. It is also interesting to compare these charts when process parameters, i.e. the target mean and standard deviation are estimated from a Phase-I dataset. In most real situations, process parameters have to be estimated as they are rarely known. Another topic for future work involves comparing the multivariate synthetic and multivariate EWMA (MEWMA) charts when the process parameters are both known and unknown. Research can also be made when the underlying process has a skewed distribution or when the independence assumption of the data is violated.

\section{APPENDIX}

TABLE A.I: ARLS AND SDRLS OF THE EWMA CHART FOR $n=3,5,7$, AND 10, wHEN $\delta_{\text {opt }}=1$, BASED ON $\mathrm{ARL}_{0}=250$

\begin{tabular}{ccccccccc}
\hline \hline$n$ & 3 & 5 & 7 & 10 & 3 & 5 & 7 & 10 \\
$\lambda$ & 0.34 & 0.48 & 0.63 & 0.78 & 0.34 & 0.48 & 0.63 & 0.78 \\
$L$ & 0.735 & 0.716 & 0.735 & 0.727 & 0.735 & 0.716 & 0.735 & 0.727 \\
\hline$\delta$ & \multicolumn{6}{c}{ ARL } & \multicolumn{6}{c}{ SDRL } \\
0.00 & 252.0 & 250.5 & 250.5 & 250.2 & 249.6 & 248.9 & 249.5 & 249.4 \\
0.30 & 52.6 & 42.7 & 40.1 & 36.3 & 49.5 & 40.5 & 38.6 & 35.2 \\
0.50 & 13.5 & 9.7 & 8.3 & 6.7 & 10.6 & 7.7 & 6.8 & 5.7 \\
0.80 & 6.2 & 4.3 & 3.4 & 2.6 & 3.8 & 2.6 & 2.2 & 1.7 \\
1.00 & 3.9 & 2.6 & 2.0 & 1.5 & 1.9 & 1.3 & 1.0 & 0.8 \\
1.50 & 2.3 & 1.5 & 1.2 & 1.0 & 0.8 & 0.6 & 0.4 & 0.2 \\
2.00 & 1.7 & 1.1 & 1.0 & 1.0 & 0.6 & 0.3 & 0.1 & 0.0 \\
3.00 & 1.1 & 1.0 & 1.0 & 1.0 & 0.3 & 0.0 & 0.0 & 0.0 \\
4.00 & 1.0 & 1.0 & 1.0 & 1.0 & 0.0 & 0.0 & 0.0 & 0.0 \\
\hline \hline
\end{tabular}

TABLE A.II: ARLS AND SDRLS OF THE SYNTHETIC CHART FOR $n=3,5$, 7, AND 10, WHEN $\delta_{\text {opt }}=1$, BASED ON $\mathrm{ARL}_{0}=250$

\begin{tabular}{ccccccccc}
\hline \hline$n$ & 3 & 5 & 7 & 10 & 3 & 5 & 7 & 10 \\
$L^{\prime}$ & 6 & 4 & 3 & 2 & 6 & 4 & 3 & 2 \\
$k$ & 2.216 & 2.139 & 2.084 & 2.002 & 2.216 & 2.139 & 2.084 & 2.002 \\
\hline$\delta$ & \multicolumn{7}{c}{ ARL } \\
\cline { 2 - 9 } 0.00 & 250.0 & 250.0 & 250.0 & 250.0 & 281.6 & 276.5 & 273.3 & 269.4 \\
0.50 & 25.9 & 13.3 & 8.4 & 5.3 & 32.5 & 16.6 & 10.3 & 6.3 \\
0.75 & 8.0 & 3.9 & 2.6 & 1.8 & 9.9 & 4.5 & 2.6 & 1.6 \\
1.00 & 3.6 & 1.9 & 1.4 & 1.2 & 3.7 & 1.6 & 0.9 & 0.5 \\
1.50 & 1.5 & 1.1 & 1.0 & 1.0 & 0.9 & 0.4 & 0.2 & 0.1 \\
2.00 & 1.1 & 1.0 & 1.0 & 1.0 & 0.4 & 0.1 & 0.0 & 0.0 \\
3.00 & 1.0 & 1.0 & 1.0 & 1.0 & 0.0 & 0.0 & 0.0 & 0.0 \\
4.00 & 1.0 & 1.0 & 1.0 & 1.0 & 0.0 & 0.0 & 0.0 & 0.0 \\
\hline \hline
\end{tabular}


TABLE A.III: ARLS OF THE EWMA AND SYNTHETIC CHARTS, WHERE $n$ $=3,5,7, \operatorname{AND} 10, \delta_{\text {opt }}=1, \mathrm{AND} \mathrm{ARL}_{0}=250$

\begin{tabular}{ccccccccc}
\hline \hline$n$ & 3 & 5 & 7 & 10 & 3 & 5 & 7 & 10 \\
\hline$\delta$ & \multicolumn{9}{c}{ EWMA } \\
\cline { 2 - 9 } 0.00 & 252.0 & 250.5 & 250.5 & 250.2 & 250.0 & 250.0 & 250.0 & 250.0 \\
0.25 & 52.6 & 42.7 & 40.1 & 36.3 & 108.1 & 74.8 & 55.8 & 39.9 \\
0.50 & 13.5 & 9.7 & 8.3 & 6.7 & 25.9 & 13.3 & 8.4 & 5.3 \\
0.75 & 6.2 & 4.3 & 3.4 & 2.6 & 8.0 & 3.9 & 2.6 & 1.8 \\
1.00 & 3.9 & 2.6 & 2.0 & 1.5 & 3.6 & 1.9 & 1.4 & 1.2 \\
1.50 & 2.3 & 1.5 & 1.2 & 1.0 & 1.5 & 1.1 & 1.0 & 1.0 \\
2.00 & 1.7 & 1.1 & 1.0 & 1.0 & 1.1 & 1.0 & 1.0 & 1.0 \\
3.00 & 1.1 & 1.0 & 1.0 & 1.0 & 1.0 & 1.0 & 1.0 & 1.0 \\
4.00 & 1.0 & 1.0 & 1.0 & 1.0 & 1.0 & 1.0 & 1.0 & 1.0 \\
\hline \hline
\end{tabular}

TABLE A.IV: SDRLS OF THE EWMA AND SYNTHETIC CHARTS, WHERE $n$ $=3,5,7$, AND 10, $\delta_{\text {opt }}=1, \mathrm{AND} \mathrm{ARL}_{0}=250$

\begin{tabular}{ccccccccc}
\hline \hline$n$ & 3 & 5 & 7 & 10 & 3 & 5 & 7 & 10 \\
\hline$\delta$ & \multicolumn{3}{c}{ EWMA } \\
\cline { 2 - 9 } 0.00 & 249.6 & 248.9 & 249.5 & 249.4 & 281.6 & 276.5 & 273.3 & 269.4 \\
0.25 & 49.5 & 40.5 & 38.6 & 35.2 & 126.8 & 87.4 & 65.0 & 46.1 \\
0.50 & 10.6 & 7.7 & 6.8 & 5.7 & 32.5 & 16.6 & 10.3 & 6.3 \\
0.75 & 3.8 & 2.6 & 2.2 & 1.7 & 9.9 & 4.5 & 2.6 & 1.6 \\
1.00 & 1.9 & 1.3 & 1.0 & 0.8 & 3.7 & 1.6 & 0.9 & 0.5 \\
1.50 & 0.8 & 0.6 & 0.4 & 0.2 & 0.9 & 0.4 & 0.2 & 0.1 \\
2.00 & 0.6 & 0.3 & 0.1 & 0.0 & 0.4 & 0.1 & 0.0 & 0.0 \\
3.00 & 0.3 & 0.0 & 0.0 & 0.0 & 0.0 & 0.0 & 0.0 & 0.0 \\
4.00 & 0.0 & 0.0 & 0.0 & 0.0 & 0.0 & 0.0 & 0.0 & 0.0 \\
\hline \hline
\end{tabular}

\section{REFERENCES}

[1] D. L. Goetsch and S. David, Quality Management for Organizational Excellence: Introduction to Total Quality, $7^{\text {th }}$ ed., Upper Saddle River, N.J.: Pearson Education, 2013, ch. 15, pp. 263.

[2] D. C. Montgomery, Statistical Quality Control: A Modern Introduction, $6^{\text {th }}$ ed., N.Y.: John Wiley \& Sons, 2009, ch. 11, p. 497.

[3] S. W. Roberts, "Control chart tests based on geometric moving averages," Technometrics, vol. 1, pp. 239-250, 1959.

[4] S. V. Crowder, "A simple method for studying run length distributions of exponentially weighted moving average charts," Technometrics, vol. 29, pp. 401-407, 1987.

[5] S. V. Crowder, "Average run lengths of exponentially weighted moving average charts," Journal of Quality Technology, vol. 19, pp. 161-164, 1987

[6] S. V. Crowder, "Design of exponentially weighted moving average schemes," Journal of Quality Technology, vol. 21, pp. 155-162, 1989.

[7] J. M. Lucas and M. S. Saccucci, "Exponentially weighted moving average control schemes: Properties and enhancements," Technometrics, vol. 32, pp. 1-12, 1990.
[8] L. Zhang, G. Chen, and P. Castagliola, "On $t$ and EWMA $t$ charts for monitoring changes in the process mean," Quality and Reliability Engineering International, vol. 25, pp. 933-945, 2009.

[9] Z. Wu and T. A. Spedding, "A synthetic control chart for detecting small shifts in the process mean," Journal of Quality Technology, vol. 32, pp. 32-38, 2000.

[10] R. B. Davis and W. H. Woodall, "Evaluating and improving the synthetic control chart," Journal of Quality Technology, vol. 34, pp. 200-208, 2002.

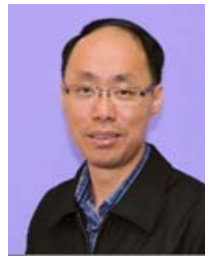

Michael B. C. Khoo is a professor in the School of Mathematical Sciences, UniversitiSains Malaysia (USM). He specializes in statistical Process control. He publishes extensively in international journals. $\mathrm{He}$ also serves as a member of the editorial boards of several international journals. His interests include statistical quality control, focusing on quality control charts. He is a member of the American Society for Quality (ASQ) and a life member of the Malaysian Mathematical Sciences Society.

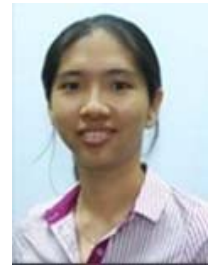

S. Y. Teh is a senior lecturer in the School of Management, UniversitiSains Malaysia (USM). She received her Ph.D. in statistical quality control from USM in 2012. She has published a few papers in international journals. She serves as a member of the editorial boards of several international journals and has reviewed numerous papers for international journals. Her interests include statistical process control, control charts, data mining, quality management, operations management and robust tests of spread.

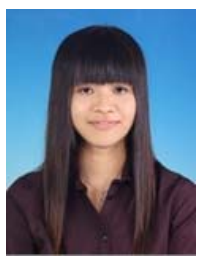

X. Y. Chew is a full time Ph.D. student in the School of Mathematical Sciences, Universiti Sains Malaysia (USM). She obtained her bachelor in information technology degree with honours (industrial computing) from Universiti Kebangsaan Malaysia in 2013.

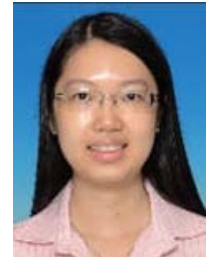

W. L. Teoh is an assistant professor in the Department of Physical and Mathematical Science, Faculty of Science, Universiti Tunku Abdul Rahman (UTAR). She received her Ph.D. in statistical quality control and her bachelor in science with education (honours) in mathematics from UniversitiSains Malaysia (USM) in 2013 and 2010 , respectively. She specializes in statistical 